Conclusion: This study suggests that JIA patients treated with DMARDs continued their treatment during the pandemic. In contrast, parents' reluctance was a major obstacle for returning to school. Therefore, more solidified school reopening strategies should be developed.

REFERENCES:

[1] Filocamo G, et al. "Absence of severe complications from SARS-CoV-2 infection in children with rheumatic diseases treated with biologic drugs." J Rheumatol. 25 avr 2020;

[2] Tagarro A, et al. "Screening and Severity of Coronavirus Disease 2019 (COVID-19) in Children in Madrid, Spain." JAMA Pediatr. 8 avr 2020;

[3] Lu X, et al. « SARS-CoV-2 Infection in Children". N Engl J Med. 23 avr 2020;382(17):1663-5.

Acknowledgements: We would like to thank all the participants involved in this clinical research and six medical students who helped completing the forms: Margaux Blondel, Alice Bonnod, Marie Desval, Béatrice Dordain, Gabrielle Fagnet, and Madouc De Saint Martin Pernot.

Disclosure of Interests: None declared

DOI: 10.1136/annrheumdis-2021-eular.2838

\section{POS1315 USEFULNESS OF SYNOVIAL BIOPSY IN THE DIFFERENTIAL DIAGNOSIS AND AS POSSIBLE PREDICTOR OF RESPONSE TO TREATMENT IN JUVENILE IDIOPATHIC ARTHRITIS}

S. Costi ${ }^{1}$, F. Pregnolato ${ }^{2}$, A. Parafioriti ${ }^{3}$, E. Armiraglio ${ }^{3}$, T. Giani ${ }^{4,5}$, R. Cimaz $^{6,7}$ ${ }^{1}$ University of Milan, Rheumatology, Milan, Italy; ${ }^{2}$ Auxologico Italiano, Experimental Laboratory of Immunological and Rheumatologic Researches, Milan, Italy; ${ }^{3}$ ASST G.Pini-CTO, Pathological Anatomy, Milan, Italy; ${ }^{4}$ Meyer Children's Hospital, Pediatric Rheumatology, Florence, Italy; ${ }^{5}$ University of Siena, Department of Medical Biotechnology, Siena, Italy; ${ }^{6}$ University of Milan, Department of Clinical Sciences and Community Health, and Research Center for Adult and Pediatric Rheumatic Diseases, Milan, Italy; ${ }^{7}$ ASST G.Pini-CTO, Pediatric Rheumatology, Milan, Italy

Background: While synovial biopsy is an invasive procedure and is not required for the diagnosis of juvenile idiopathic arthritis (JIA), it may be useful in doubtful cases.

Objectives: Aims of the study were i.to verify the role of synovial biopsy in the differential diagnosis of JIA and ii. to review the pathology slides in order to evaluate possible associations of a histologic pattern with response to treatment.

Methods: We reviewed data from medical records of patients under the age of 18 years who underwent a synovial biopsy requested by our Pediatric Rheumatology Unit over the last 10 years. We collected information on demographic, clinical, laboratory, radiological, histopathological characteristics, as well as treatment response (in particular, remission at the last visit and number of examination, number of biologic drugs used). Among variables in the histologic score, number of layers in the synovial lining and inflammatory infiltrate (0-5) were compared to clinical status at last visit. Potential differences in variables between responders and non responders were assessed by unpaired t-test or non-parametric Mann-Whitney test, as appropriate.

Results: We identified 64 patients $(40 \mathrm{~F}, 24 \mathrm{M})$ with a median age at disease onset of 9 years (range 1-15) and a median follow-up time of 161 months (range 8-1160). We recognized two groups of interest: patients with a known JIA diagnosis (28/64) and patients with unknown diagnosis (36/64) at the moment of synovial biopsy. In the group with known JIA, most underwent the procedure during orthopedic surgery, and in all cases the histology was consistent with JIA. Among the unknown diagnosis group, in 19 cases results were consistent with a chronic synovitis, while among the other 17 histology could lead to a diagnosis of other conditions in 6 cases (foreign body and villonodular synovitis $n=2$ each, sarcoidosis and osteochondromatosis $n=1$ each). In the remaining 11 the final diagnoses were varied (mostly genetic forms eg skeletal dysplasia, CACP, Thiemann disease).

Between the two groups we identified 46 patients with a definite JIA diagnosis. At the last follow-up visit 29 of them were in clinical remission, albeit on medication. The remaining 17 had a severe course of disease, with persistent activity and use of at least two biologic drugs. In 26 cases we could evaluate the correlation between status at last visit and number of layers/inflammatory infiltrate, but no statistical significant correlation was found

Conclusion: Despite its limited use nowadays, synovial biopsy may still be a useful tool in patients whose diagnosis is unclear. In our study, while it confirmed the suspicion in most cases, in other instances it allowed the diagnosis of rare conditions that would have been otherwise missed. No association between disease course and histological features in a small JIA cohort was found. We are currently expanding the study with a larger series.

Disclosure of Interests: None declared

DOI: 10.1136/annrheumdis-2021-eular.2989

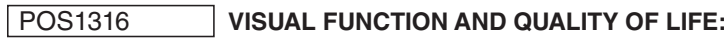 PRELIMINARY RESULTS FROM A PIVOTAL CROSS SECTIONAL STUDY ON ONE HUNDRED PATIENTS WITH JUVENILE IDIOPATHIC ARTHRITIS-ASSOCIATED AND IDIOPATHIC UVEITIS}

G. B. Beretta ${ }^{1}$, F. Minoia ${ }^{1}$, L. Marelli ${ }^{2}$, C. Mapelli ${ }^{3}$, G. Leone ${ }^{3}$, T. Giani ${ }^{4}$, P. Nucci ${ }^{2,5}$, E. Miserocchi ${ }^{6}$, R. Cimaz ${ }^{7,8}$ on behalf of Pediatric Rheumatology Associated Group of the Milan Area. ${ }^{1}$ Fondazione IRCCS Ca' Granda Ospedale Maggiore Policlinico, Pediatric Rheumatology, Milan, Italy; ${ }^{2}$ Ospedale San Giuseppe, IRCCS Multimedica, Ophthalmology, Milan, Italy; ${ }^{3}$ Fondazione IRCCS Ca' Granda Ospedale Maggiore Policlinico, Ophthalmology, Milan, Italy; ${ }^{4}$ Università degli Studi di Siena, Pediatric Rheumatology, Siena, Italy; ${ }^{5}$ Università degli Studi di Milano, Ophthalmology, Milan, Italy; ${ }^{6}$ IRCCS Ospedale San Raffaele, Ophthalmology, Milan, Italy; ${ }^{7}$ ASST Gaetano Pini-CTO, Milan, Italy, Pediatric Rheumatology, Milan, Italy; ${ }^{8}$ Università degli Studi di Milano, Pediatric Rheumatology, Milan, Italy

Background: Juvenile idiopathic arthritis (JIA) is the main cause of chronic uveitis in childhood and JIA associated uveitis (JIA-U) is the most common extraarticular complication of JIA. Despite continuous improvement in its management, pediatric uveitis still represents a serious condition with potential sight-threatening complications and a significant impact on quality of life (QoL).

Objectives: To evaluate visual function (VF) and QoL in children with JIA-U and idiopathic uveitis

Methods: A cross-sectional study was conducted in two tertiary Pediatric Rheumatology Centres, enrolling all patients seen with JIA-U, JIA without uveitis and idiopathic uveitis. VF was assessed by a translated form of the available EYE$Q$, adapted for cross-cultural feasibility into a 10-question tool, while $Q$ oL was evaluated by the Italian version of the Pediatric Rheumatology Quality of Life scale part of the Juvenile Arthritis Multidimensional Report (JAMAR), shortened for feasibility to a 8-question tool. JAMAR section on treatment compliance and school attendance was also included. Parents, and patients when appropriate, were asked to complete each patient/parent-reporting outcome measure answering on a 4-point Likert scale, with a total score ranging from 0 to 72 (worst condition). Medical charts were reviewed regarding JIA and uveitis features and outcome. Quantitative and qualitative variables were compared by means of Mann-Whitney $U$ test or chi-square/Fisher exact test, as appropriate; correlations among quantitative non-parametric variables were evaluated by Spearman's test.

Results: We herein describe results from the first 100 patients enrolled $(76 \%$ female), with a median age at study time of 12.8 (9.0-17.6) years. Forty-nine had JIA-U, 37 JIA without uveitis and 14 idiopathic uveitis. Uveitis was active in $14 / 63$ patients $(22.2 \%)$, with a median of uveitis duration of 9.0 years (3.614.8). Almost all children with uveitis were on systemic treatment $(58 / 63,92 \%)$ at the time of interview; $54.0 \%$ of patients presented an ocular damage, with $8.0 \%$ having a best corrected visual acuity $(B C V A)<4 / 10$. Total score, VF and QoL scores resulted significantly higher in JIA-U patients compared to JIA without uveitis, while no differences were noticed among children with uveitis with or without JIA (Table 1). School absence was reported more frequently in JIA-U compared to JIA only ( $32.7 \%$ vs $10.8 \%$, p 0.0211$)$. VF was significantly worse in patients with ocular damage and BCVA $<4 / 10$ ( $p 0.0351$ and 0.0123 , respectively). In patients with uveitis, VF and QoL showed a significant correlation ( $r$ $0.50, p<0.0001)$ especially in patients with idiopathic uveitis ( $r 0.74, p<0.0001)$. Conclusion: Visual function is a crucial component of QoL in children with uveitis and it correlates with ocular damage. Since eye involvement significantly affect QoL in patients with JIA, a specific tool widely validated and cross-cultural adapted is highly demanded in the clinical care of JIA-U patients.

\section{REFERENCES:}

[1] Angeles-Han ST et al. The importance of visual function in the quality of life of children with uveitis. J AAPOS, 2010. Filocamo et al. A New Approach to Clinical Care of Juvenile Idiopathic Arthritis: The Juvenile Arthritis Multidimensional Assessment Report. J Rheumatol, 2011. 